\title{
DISTANCE-DEPENDING ELECTRON-PHONON INTERACTIONS FROM ONE- AND TWO-BODY ELECTRONIC TERMS IN A DIMER.
}

\author{
M. ACQUARONE \\ C.N.R.-G.N.S.M., Unita' I.N.F.M., Dipartimento di Fisica,Universita' di Parma \\ Parma, I-43100, Italy \\ and \\ C. NOCE \\ Unità I.N.F.M., Dipartimento di Scienze Fisiche "E.R. Caianiello", Università di Salerno, \\ Baronissi, I-84081, Italy
}

\begin{abstract}
.
For a dimer with a non-degenerate orbital built from atomic wave functions of Gaussian shape we evaluate all the electron-phonon couplings derived from the one-body and two-body electronic interactions, considering both the adiabatic and extreme non-adiabatic limit. Not only the values of the coupling parameters in the two limits, but also the expressions of the corresponding terms in the Hamiltonian differ. Depending on the distance between the dimer ions, some of the two-body couplings are comparable, or even larger than the one-body ones
\end{abstract}

\section{The Model.}

In a general two-site electron-phonon Hamiltonian $H=H_{e l}+H_{p h}+H_{e l-p h}$ the interacting term $H_{e l-p h}$ originates from developing $H_{e l}$ to first order in the ion displacements $u_{i} \quad(i=1,2)$, where, in standard notation for a non-degenerate orbital:

$$
\begin{gathered}
H_{e l}=\epsilon \sum_{\sigma}\left(n_{1 \sigma}+n_{2 \sigma}\right)+\sum_{\sigma}\left[t+X\left(n_{1-\sigma}+n_{2-\sigma}\right)\right]\left(c_{1 \sigma}^{\dagger} c_{2 \sigma}+H . c .\right)+U\left(n_{1 \uparrow} n_{1 \downarrow}+n_{2 \uparrow} n_{2 \downarrow}\right) \\
+(V-J / 2) n_{1} n_{2}-2 J\left[S_{1}^{z} S_{2}^{z}+\frac{1}{2}\left(S_{1}^{+} S_{2}^{-}+H . c .\right)\right]+P\left(c_{1 \uparrow}^{\dagger} c_{1 \downarrow}^{\dagger} c_{2 \downarrow} c_{2 \uparrow}+H . c .\right) .
\end{gathered}
$$

We shall develop both the one- $(\epsilon, t)$ and two-body $\left(X, U, V, J_{z}=J_{x y}=P\right)$ electron interaction parameters, evaluated as in Ref.1, by assuming a nondegenerate orbital described by Wannier functions built from atomic orbitals of Gaussian shape. We associate to each site a Gaussian atomic-like orbital $\phi_{i}(\mathbf{r}-$ $\left.\mathbf{R}_{\mathbf{i}}\right)$, with the ions centered at the positions $\mathbf{R}_{\mathbf{i}} \equiv\left( \pm \mathbf{a} / \mathbf{2}+\mathbf{u}_{\mathbf{i}}, \mathbf{0}, \mathbf{0}\right) \quad(\mathbf{i}=\mathbf{1}, \mathbf{2})$. By defining $N \equiv(2 / \pi)^{3 / 4} \Gamma^{3 / 2}$, they read: $\phi_{i}(\mathbf{r})=\mathbf{N} \exp \left\{-\boldsymbol{\Gamma}^{\mathbf{2}}\left[\left(\mathbf{x} \pm \mathbf{a} / \mathbf{2}-\mathbf{u}_{\mathbf{i}}\right)^{\mathbf{2}}+\mathbf{y}^{\mathbf{2}}+\mathbf{z}^{\mathbf{2}}\right]\right\}$. Then the Wannier functions $\Psi_{1}, \Psi_{2}$ can be written as:

$$
\Psi_{1}(\mathbf{r})=\mathbf{A}(\mathbf{S}) \phi_{\mathbf{1}}(\mathbf{r})+\mathbf{B}(\mathbf{S}) \phi_{\mathbf{2}}(\mathbf{r}) \quad \boldsymbol{\Psi}_{\mathbf{2}}(\mathbf{r})=\mathbf{B}(\mathbf{S}) \phi_{\mathbf{1}}(\mathbf{r})+\mathbf{A}(\mathbf{S}) \phi_{\mathbf{2}}(\mathbf{r})
$$


$A(S)=\left[(1+S)^{-1 / 2}+(1-S)^{-1 / 2}\right] / 2 \quad B(S)=\left[(1+S)^{-1 / 2}-(1-S)^{-1 / 2}\right] / 2$

with $S(u) \equiv\left\langle\phi_{1} \mid \phi_{2}\right\rangle=\exp \left[-\Gamma^{2}(a+u)^{2} / 2\right]$, and $u=u_{2}-u_{1}$. To make clear our method of calculation, it is convenient to explicitate, as an example, the one-body local electronic energy:

$\epsilon^{(i)}=\int \Psi_{i}^{*}\left(\mathbf{r}, \mathbf{R}_{\mathbf{1}}, \mathbf{R}_{\mathbf{2}}\right)\left[-\frac{\mathbf{h}^{2}}{\mathbf{2} \mathbf{m}} \nabla^{2}+\mathbf{V}_{\mathbf{1}}\left(\mathbf{r}-\mathbf{R}_{\mathbf{1}}\right)+\mathbf{V}_{\mathbf{2}}\left(\mathbf{r}-\mathbf{R}_{\mathbf{2}}\right)\right] \Psi_{\mathbf{i}}\left(\mathbf{r}, \mathbf{R}_{\mathbf{1}}, \mathbf{R}_{\mathbf{2}}\right) \mathbf{d}^{3} \mathbf{r}$

where the potentials originating from the ion cores at the displaced positions $\mathbf{R}_{1}$ and $\mathbf{R}_{2}$ are:

$$
V_{i} \equiv V\left(\mathbf{r}-\mathbf{R}_{\mathbf{i}}\right)=-\mathbf{e}^{2} \mathbf{Z}\left[\left(\mathbf{x} \pm \mathbf{a} / \mathbf{2}-\mathbf{u}_{\mathbf{i}}\right)^{2}+\mathbf{y}^{2}+\mathbf{z}^{2}\right]^{-\mathbf{1} / \mathbf{2}} \quad(\mathbf{i}=\mathbf{1}, \mathbf{2})
$$

with $-e$ the electron charge, and $+Z e$ the charge of the ion core. The local energy $\epsilon$ (actually site-independent) can be decomposed into three terms, corresponding to the contributions from the kinetic operator $\left(\epsilon_{\nabla}^{(i)}\right)$ and from each one of the ionic potentials $\left(\epsilon_{V_{1}}^{(i)}, \epsilon_{V_{2}}^{(i)}\right.$, respectively). A similar decomposition holds for the hopping amplitude $t$.

We need to distinguish between the adiabatic and the non-adiabatic limit in evaluating the electron-phonon interactions, because the integrals have different kernels in the two cases. Indeed, in the adiabatic limit the displacements affect both the potentials $V\left(\mathbf{r}-\mathbf{R}_{\mathbf{i}}\right)$ and the Wannier functions $\Psi_{i}\left(\mathbf{r}, \mathbf{R}_{\mathbf{1}}, \mathbf{R}_{\mathbf{2}}\right)$ , expressing the requisite that the electronic charge distribution adjusts itself instantaneously at the position of the ions. We shall schematize the opposite situation, where the electrons are slower than the ions, as realized by the electronic charge distribution staying centred around the undisplaced ion position, while the potentials are centred on the displaced ions. We shall call this the extreme anti-adiabatic limit. In the literature[2 the two limits are also named from, respectively, Frölich and Bloch.

\section{Coupling terms in the anti-adiabatic limit.}

In the anti-adiabatic limit $\epsilon_{\nabla}$ does not change, therefore no electron-phonon coupling originates from it. The coupling terms derived from the two-body interactions are also identically vanishing in this limit, because they involve the Wannier functions and the inter-electronic Coulomb potential which are both insensitive to the displacements of the ions. The only non-vanishing electronphonon non-adiabatic interactions arise from the variation of the potential contributions to $\epsilon$ and $t$. Let us now succinctly describe their evaluation. Full details are given in Ref.3.

The perturbation of $\epsilon_{V_{i}}^{(i)}$ originates a term in the Hamiltonian connecting the local charge with the local deformation: $\sum_{\sigma}\left(g_{0}^{(1)} n_{1 \sigma} u_{1}+g_{0}^{(2)} n_{2 \sigma} u_{2}\right)$. It has a formal similarity with the Holstein coupling term[ [4], but it has a physically different origin, as Holstein [4] considered electrons moving along a chain of fixed spacing with vibrating diatomic molecules at its nodes. On site 1, its explicit 
evaluation [3] yields:

$$
g_{0}^{(1)}=\frac{2 \Gamma \sqrt{2 / \pi}}{a}\left\{B^{2}\left[F_{0}\left(2 a^{2} \Gamma^{2}\right)-S^{4}\right]+4 A B S\left[F_{0}\left(\frac{a^{2} \Gamma^{2}}{2}\right)-S\right]\right\} .
$$

where $F_{0}(x)=x^{-1 / 2} \operatorname{Erf}\left(x^{1 / 2}\right)$. As, under site permutation, $a \rightarrow-a, g_{0}^{(1)}=$ $-g_{0}^{(2)}$, as might have been anticipated by considering that, for equal charges $n_{1}=n_{2}$ and displacement amplitudes, with $\mathbf{e}_{12} \equiv\left(\mathbf{R}_{\mathbf{2}}-\mathbf{R}_{\mathbf{1}}\right) / \mathbf{a}$, the energies $g_{0}^{(1)} n_{1} \mathbf{u}_{1} \bullet \mathbf{e}_{12}$ and $g_{0}^{(2)} n_{2} \mathbf{u}_{2} \bullet \mathbf{e}_{12}$ on both sites coincide. Now symmetry requires $u_{1}=-u_{2}$ (a constraint which does not hold for the original Holstein model [4]) from which $g_{0}^{(2)}=-g_{0}^{(1)}$ follows. The contribution to the Hamiltonian then reads: $g_{0}^{(1)} \sum_{\sigma}\left(n_{1 \sigma} u_{1}-n_{2 \sigma} u_{2}\right)$

The "crystal-field" coupling term $g_{c f}^{(i j)}$ expresses the change in the energy $\epsilon_{V_{j}}^{(i)}$. To establish the form of this term, let us consider site 1 , with charge $n_{1}$. Its energy, after a displacement $\mathbf{u}_{2}$ of the ion on site 2, changes by an amount $E^{(1)}=g_{c f}^{(12)} n_{1} \mathbf{u}_{2} \bullet \mathbf{e}_{12}$. This can be considered as the quantity measured by an observer sitting on ion 1 and watching the ion 2 moved by $\mathbf{u}_{2}$. The equivalent measurement done by an observer on ion 2 watching the ion 1 displaced by $\mathbf{u}_{1}$, yields $E^{(2)}=g_{c f}^{(21)} n_{2} \mathbf{u}_{1} \bullet \mathbf{e}_{12}$. Assuming $n_{1}=n_{2}$, and $u_{1}=-u_{2}$ one must have $E^{(1)}=E^{(2)}$, implying $g_{c f}^{(12)}=-g_{c f}^{(21)}$. Therefore for the dimer as a whole one writes this term as $g_{c f}^{12} \sum_{\sigma}\left(n_{1 \sigma} u_{2}-n_{2 \sigma} u_{1}\right)$. The explicit evaluation[3] for site 1 yields:

$$
g_{c f}^{(12)}=-2 A\left(\frac{\Gamma}{a}\right) \sqrt{\frac{2}{\pi}}\left[A F_{0}\left(2 a^{2} \Gamma^{2}\right)+4 B S F_{0}\left(a^{2} \Gamma^{2} / 2\right)-4 B S^{2}-A S^{4}\right] .
$$

From Eq.6 and the change of sign of $a$ under site permutation, $g^{(12)}=-g^{(21)}$ follows.

The Su-Schrieffer-Heeger Hamiltonian $H_{S S H} \equiv \gamma^{(12)} \sum_{\sigma}\left(c_{1 \sigma}^{\dagger} c_{2 \sigma}+c_{2 \sigma}^{\dagger} c_{1 \sigma}\right)\left(u_{2}-u_{1}\right)$, is due to the modulation of the hopping amplitude $t$. To preserve the invariance of $H_{S S H}$ under site permutation, $\gamma^{(12)}=-\gamma^{(21)}$ (see e.g refs.6 and 7). Indeed, its explicit expression[3] is:

$$
\begin{gathered}
\gamma^{(12)}=4 \sqrt{\frac{2}{\pi}}\left(\frac{\Gamma}{a}\right)\left\{\frac{A B}{2}\left[S_{0}^{4}-\left(1-4 a^{2} \Gamma^{2}\right) F_{0}\left(2 a^{2} \Gamma^{2}\right)\right]\right\} \\
+4 \sqrt{\frac{2}{\pi}}\left(\frac{\Gamma}{a}\right)\left\{\left(A^{2}+B^{2}\right) S_{0}\left[S_{0}-F_{0}\left(a^{2} \Gamma^{2} / 2\right)\right]\right\} .
\end{gathered}
$$

Under site permutation $a \rightarrow-a$ and $A \rightarrow B$ so that $\gamma^{(12)}=-\gamma^{(21)}$ as expected.

In conclusion, in the non-adiabatic limit the complete electron-phonon Hamiltonian is given by:

$$
\begin{gathered}
H_{e p}^{n a}=g_{0}^{(1)} \sum_{\sigma}\left(n_{1 \sigma} u_{1}-n_{2 \sigma} u_{2}\right)+g_{c f}^{(12)} \sum_{\sigma}\left(n_{1 \sigma} u_{2}-n_{2 \sigma} u_{1}\right) \\
+\gamma^{(12)} \sum_{\sigma}\left(c_{1 \sigma}^{\dagger} c_{2 \sigma}+c_{2 \sigma}^{\dagger} c_{1 \sigma}\right)\left(u_{2}-u_{1}\right)
\end{gathered}
$$




\section{Coupling terms in the adiabatic limit.}

In the explicit expression of the different electronic interactions in the adiabatically displaced state, $u$ invariably enters in the combination $a+u$. So, to obtain the corresponding couplings, one can simply take the derivative with respect to $a$ of the parameters in Eq.1 as evaluated in Ref.1.

There is some confusion in the literature about the correct form of the electron-phonon Hamiltonian obtained, in the adiabatic limit, from the variation of the local energy $\epsilon$, therefore we shall devote some space to clarify this point. In this limit, $u_{i} \neq 0$ enters both the charge distributions and the potentials. The "crystal field" interaction couples the charge on site $i$ to the relative position of site $j$ through the modification of both the kinetic and the potential contributions to $\epsilon^{(i)}$. Let's place the origin of the $x$-coordinate onto one of the displaced ions, at $\mathbf{R}_{\mathbf{i}}$, say. One has then to take into account the relative displacement of the ions. In the adiabatic limit, therefore, the overall $\epsilon$-derived electron-phonon coupling term in the Hamiltonian is: $g_{\epsilon}^{(12)} \sum_{\sigma} n_{1 \sigma}\left(u_{2}-u_{1}\right)+g_{\epsilon}^{(21)} \sum_{\sigma} n_{2 \sigma}\left(u_{1}-u_{2}\right)$. As $g_{\epsilon}^{(12)}=-g_{\epsilon}^{(21)}$ we can write the total adiabatic contribution from local energy terms to the electronphonon Hamiltonian as $H_{e p}^{\epsilon}=g_{\epsilon}^{(12)} \sum_{\sigma}\left(n_{2 \sigma}+n_{1 \sigma}\right)\left(u_{2}-u_{1}\right)$. A Hamiltonian of this form was used in the papers of Ref.8, while those of Ref.9 proposed Hamiltonians incompatible with our results. After including the $S S H$ term, the complete one-body electron-phonon Hamiltonian in the adiabatic limit has therefore the form 10:

$$
H_{e p}^{a d}=g_{\epsilon}^{(12)} \sum_{\sigma}\left(n_{1 \sigma}+n_{2 \sigma}\right)\left(u_{2}-u_{1}\right)+\gamma^{(12)} \sum_{\sigma}\left(c_{1 \sigma}^{\dagger} c_{2 \sigma}+c_{2 \sigma}^{\dagger} c_{1 \sigma}\right)\left(u_{2}-u_{1}\right) .
$$

Coming now to the explicit expressions of the one-body coupling parameters, let us write for conveniency $g_{\epsilon}^{(12)} \equiv g_{\nabla}^{(12)}+g_{V}^{(12)}$ and $\gamma^{(12)} \equiv \gamma_{\nabla}^{(12)}+\gamma_{V}^{(12)}$. We obtain:

$$
\begin{gathered}
g_{\nabla}^{(12)}=-\frac{\hbar^{2}}{2 m}\left[\frac{a \Gamma^{4} S^{2}}{\left(1-S^{2}\right)^{2}}\right]\left[2\left(1-a^{2} \Gamma^{2}-S^{2}\right)\right] \\
g_{V}^{(12)}=-Z e^{2}\left(2 \Gamma \sqrt{\frac{2}{\pi}}\right)\left[\frac{\partial\left(A^{2}+B^{2}\right)}{\partial u}+\frac{4 A B S^{2}+\left(A^{2}+B^{2}\right) S^{4}}{a}\right] \\
\left.-Z e^{2}\left(2 \Gamma \sqrt{\frac{2}{\pi}}\right)\left\{F_{0}\left(2 a^{2} \Gamma^{2}\right)\left[\frac{\partial\left(A^{2}+B^{2}\right)}{\partial u}-\frac{\left(A^{2}+B^{2}\right)}{a}\right]+(4 S)\left[\frac{\partial A B}{\partial u}-\frac{A B}{a}\left(1+a^{2} \Gamma^{2}\right)\right)\right]\right\} . \\
\gamma_{\nabla}^{(12)}=\frac{\hbar^{2}}{2 m}\left[\frac{a S \Gamma^{4}}{\left(1-S^{2}\right)^{2}}\right]\left[2\left(1-S^{2}\right)-a^{2} \Gamma^{2}\left(1+S^{2}\right)\right] \\
\gamma_{V}^{(12)}=-Z e^{2}\left(4 \Gamma \sqrt{\frac{2}{\pi}}\right)\left\{\left[\frac{\partial A B}{\partial u}+\frac{A^{2}+B^{2}}{a} S^{2}+\frac{A B}{a} S^{4}\right]+\left[\frac{\partial A B}{\partial u}-\frac{A B}{a}\right] F_{0}\left(2 a^{2} \Gamma^{2}\right)\right. \\
\left.+S\left[\frac{\partial\left(A^{2}+B^{2}\right)}{\partial u}-\frac{A^{2}+B^{2}}{a}\left(1+a^{2} \Gamma^{2}\right)\right] F_{0}\left(a^{2} \Gamma^{2} / 2\right)\right\} .
\end{gathered}
$$


Notice that, as the partial derivatives are linear in $a$, they change sign under site permutation.

Also the two-body electronic interactions $U, V, J(=P), X$ of Eq. (1) give rise to electron-phonon couplings, all of the form:

$$
H_{Y}^{e p}=\left(\frac{d Y}{d a}\right) F\left(c_{i \sigma}^{\dagger}, c_{j \sigma}\right)\left(u_{j}-u_{i}\right) \quad(i, j=1,2)
$$

where $Y=U, V, X, J$ and $F\left(c_{i \sigma}^{\dagger}, c_{j \sigma}\right)$ is the function of Fermi operators representing the two-body interaction whose amplitude is $Y$. From the results of Ref.1, their evaluation is trivial. Notice that, as $U(a)=J(a)+e^{2} \Gamma / \sqrt{\pi}$, then $d U / d a=d J / d a=d P / d a$. We list below their explicit expressions:

$$
\begin{gathered}
\frac{d X}{d a}=-e^{2} \frac{\Gamma}{\sqrt{\pi}}\left[(-a \Gamma S) \frac{\left(1+3 S^{2}\right)}{\left(1-S^{2}\right)^{3}}\right]\left[1+2 S^{2}+F_{0}\left(a^{2} \Gamma^{2}\right)-2\left(1+S^{2}\right) F_{0}\left(\frac{\left.a^{2} \Gamma^{2}\right)}{4}\right)\right] \\
-e^{2} \frac{\Gamma}{\sqrt{\pi}}\left[\frac{S / a}{\left(1-S^{2}\right)^{2}}\right]\left\{4 a^{2} \Gamma^{2} S^{2}\left[F_{0}\left(\frac{a^{2} \Gamma^{2}}{4}\right)-1\right]+S^{2}-F_{0}\left(a^{2} \Gamma^{2}\right)\right\} \\
\quad-e^{2} \frac{\Gamma}{\sqrt{\pi}}\left[\frac{S / a}{\left(1-S^{2}\right)^{2}}\right]\left\{2\left(1+S^{2}\right)\left[F_{0}\left(\frac{a^{2} \Gamma^{2}}{4}\right)-\sqrt{S}\right]\right\}, \\
\frac{d U}{d a}=e^{2} \frac{\Gamma}{\sqrt{\pi}}\left[\frac{-4 a \Gamma^{2} S^{2}}{\left(1-S^{2}\right)^{3}}\right]\left[2-S^{2}+2 S^{4}+S^{2} F_{0}\left(a^{2} \Gamma^{2}\right)-4 S^{2} F_{0}\left(\frac{a^{2} \Gamma^{2}}{4}\right)\right] \\
+e^{2} \frac{\Gamma}{\sqrt{\pi}}\left[\frac{S^{2} / a}{\left(1-S^{2}\right)^{2}}\right]\left\{2 a^{2} \Gamma^{2}\left[1-4 S^{2}-F_{0}\left(a^{2} \Gamma^{2}\right)+4 F_{0}\left(\frac{a^{2} \Gamma^{2}}{4}\right)\right]\right. \\
\quad \frac{d\left(V-S^{2}-F_{0}\left(a^{2} \Gamma^{2}\right)+4\left[F_{0}\left(\frac{a^{2} \Gamma^{2}}{4}\right)-\sqrt{S}\right]\right\},}{d a}= \\
e^{2} \frac{\Gamma}{\sqrt{\pi}}\left[-\frac{4 a \Gamma^{2} S^{2}}{\left(1-S^{2}\right)^{3}}\right]\left[3-S^{2}-8 S^{4}-\left(7-5 S^{2}\right) F_{0}\left(a^{2} \Gamma^{2}\right)-4\left(1-3 S^{2}\right) F_{0}\left(\frac{a^{2} \Gamma^{2}}{4}\right)\right] \\
+e^{2} \frac{\Gamma}{\sqrt{\pi}}\left[\frac{1 / a}{\left(1-S^{2}\right)^{2}}\right]\left\{2 a^{2} \Gamma^{2} S^{2}\left[-1-4 S^{2}+F_{0}\left(a^{2} \Gamma^{2}\right)+4 F_{0}\left(\frac{a^{2} \Gamma^{2}}{4}\right)\right]\right. \\
+2 S^{2}+\frac{3}{2} S^{4}-\left(2-\frac{3}{2} S^{2}\right) F_{0}\left(a^{2} \Gamma^{2}\right)+2 S^{2}\left[F_{0}\left(\frac{a^{2} \Gamma^{2}}{4}\right)-\sqrt{S}\right\},
\end{gathered}
$$

Fig. 1 and 2 present the values of the one-body coupling constant for, respectively, the non- adiabatic, and the adiabatic limit, evaluated by assuming for the shape-controlling parameter of the Wannier functions $\Gamma$ the typical[1] value $1 . \AA^{-1}$. The most unexpected result concerns $g_{c f}$. While usually neglected in the literature[7] on metallic systems, this coupling has been recognized as relevant to polar materials [1]. We find indeed that, when $\Gamma=1.0 \AA^{-1}, g_{c f}$ is larger 


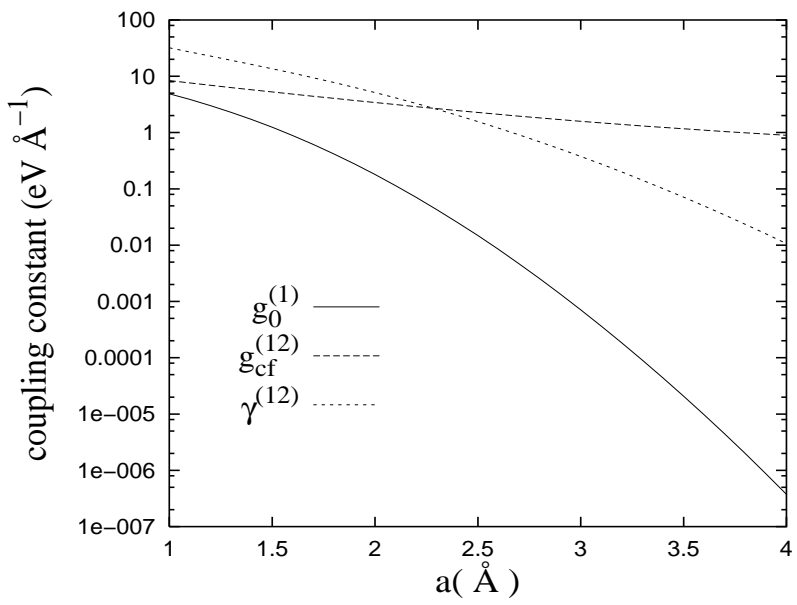

Figure 1: Non-adiabatic coupling constants $g_{0}^{(1)}, g_{c f}^{(12)}, \gamma^{(12)}\left(\right.$ in $\mathrm{eV} \AA^{-1}$ ) versus the dimer length (in $\AA$ ), evaluated assuming $\Gamma=1.0 \AA^{-1}$.

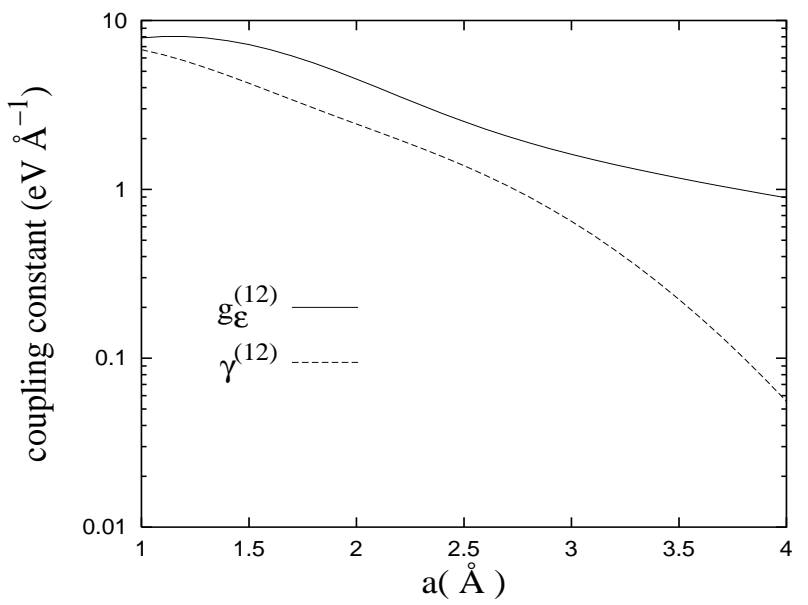

Figure 2: Adiabatic coupling constants $g_{\epsilon}^{(12)}, \gamma^{(12)}$ (in $\mathrm{eV} \AA^{-1}$ ) versus the dimer length (in $\AA$ ), for $\Gamma=1.0 \AA^{-1}$. 


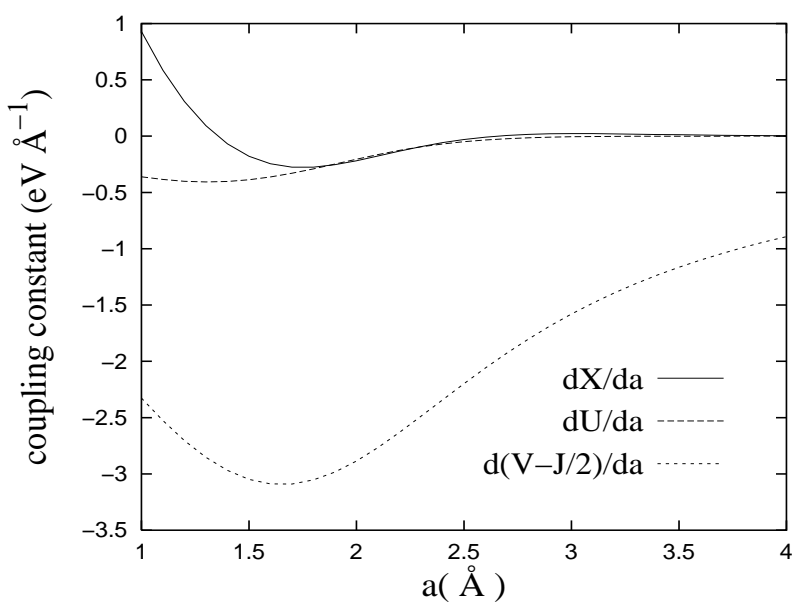

Figure 3: Adiabatic coupling constants from two-body interactions $d X / d a$, $d U / d a, d(V-J / 2) / d a\left(\right.$ in $\left.\mathrm{eV} \AA^{-1}\right)$ versus the dimer length (in $\AA$ ), for $\Gamma=1.0$ $\AA^{-1}$.

than $g_{0}$ for any $a$, and it becomes the largest parameter for $a>2.2 \AA$. For small $a$, the $S S H$ coupling is the largest.

Fig. 2 for the adiabatic case shows that $g_{\epsilon}^{(12)}$ is always larger than the $S S H$ interaction $\gamma^{(12)}$, and particularly for large $a$ there is an order of magnitude difference between them. The case of $\Gamma=2 . \AA^{-1}$ (discussed in ref.3) shows that, the more localized are the orbitals, the more relevant is the role of $g_{c f}$ in relation to the other admissible couplings. Fig. 3 shows the couplings derived from the two-body electronic interactions for the same parameters as Fig.2. In general, their values are much smaller than those of $g_{\epsilon}$ and $\gamma^{(12)}$, with the possible exception of $d(V-J / 2) / d a$. Indeed, that coupling arises from a physical mechanism not very different from the one originating $g_{V_{j}}^{(i)}$, i.e. the vibration of the charge on site $j$ as felt by site $i$. Similarly to $g_{\epsilon}$ also $d\left(V-J_{z} / 2\right) / d a$ decreases slowly with $a$, so that for large $a$ those two are the only relevant couplings. Such interactions in the lattice have been recently discussed in ref.12, while their effects in the optical spectra have been treated in ref.13.

\section{Conclusions}

We have presented the analytical evaluation of the electron-phonon coupling parameters derived from both one- and two-body electronic interactions in a model of a dimer with a non-degenerate orbital built from atomic orbital of Gaussian shape. We have shown that the coupling terms in the adiabatic and the anti-adiabatic limits differ qualitatively.

The evaluation of the coupling terms originating from the two-body electronic interactions shows that at least the one generated by the Coulomb repulsion between the charges on different sites, is comparable to, or even larger than, the couplings derived from the one-body interactions. The quantitative results for the coupling parameters, even if agreeing in order of magnitude with some estimates from experimental data 14 are obviously model-depending. However, 
their ratios should be more close to the reality. In particular, the obtained values of the coupling terms, when compared to the values of the electronic interactions resulting from the same Wannier functions 11 suggests that, for dimer lengths comparable to the lattice parameters in high temperature superconductors and colossal magnetoresistance materials, at most $d U / d a$ and $d X / d a$ can be safely dropped, while neglecting any of the other electron-phonon interactions is a questionable approximation.

Acknowledgements

It is a pleasure to thank J.R. Iglesias, M. A. Gusmão, M. Cococcioni, A. Alexandrov, and particularly A.A. Aligia and A. Painelli, for critical discussions and comments. This work was supported by I.N.F.M. and by MURST 1997 cofunded project "Magnetic Polarons in Manganites".

\section{References}

[1] M. Acquarone, J. R. Iglesias, M.A. Gusmão, C. Noce and A. Romano, Phys. Rev. B58, 7626 (1998).

[2] J. Ashkenazi, M. Dacorogna and M. Peter Solid. St. Comm. 29, 181 (1979).

[3] M. Acquarone and C. Noce, Int. J. Mod. Phys. B 13, 3331 (1999).

[4] T. Holstein, Annals of Physics 8, 325 (1959).

[5] W.P. Su, J.R. Schrieffer and A.J. Heeger, Phys. Rev. B22, 2099 (1980).

[6] S. Barisic, J.Labbé and J. Friedel, Phys. Rev. Letters 25,919 (1970).

[7] S. Barisic, Phys. Rev. B5, 932 (1972).

[8] B. K. Chakraverty, M. J. Sienko and J. Bonnerot, Phys. Rev. B17, 3781 (1978); C. A. Balseiro and L. M. Falicov,Phys. Rev. B20, 4457 (1979).

[9] A.N. Das and S. Sil J.Phys. Cond. Matter 5, 8265 (1993); A.N. Das and P. Choudhury Phys. Rev. B49, 13219 (1994); T. Sakai, D. Poilblanc and D.J. Scalapino Phys. Rev.B55, 8445 (1997).

[10] The Hamiltonians of Eqs. 8 and 9 differ from the corresponding results in Ref.3, because here we correct some mistakes which we found in that paper after it was published.

[11] S. Barisic, Europhys. Lett. 8, 765 (1989); S. Barisic, Int. J. Mod. Phys. B 5, 2439 (1991).

[12] G. D. Mahan and L. M. Woods, Phys. Rev. B60, 5276 (1999).

[13] M. Meneghetti Phys. Rev. B60, 15472 (1999).

[14] R. Hlubina and G.K.Sadiek Phys. Rev. B55, 2733 (1997). 